\title{
Index rerum ad Vol. 13
}

Confecit Wilhelm Baumann, Zurich

Acute hypotonic hyperhydration, 14 Anastomosis uretero-ureteralis, 193 Anuria obstructiva, 213

- $\quad$ osmatica, 14

Burens Disease, 1

Calculos oxalicos, 370

Galculosis renalis et hyperparathyreoi-

dismus, 294, 317 Carcinoma prostatae, 74, 144

- $\quad$ renalis, 262, 333Cavernosogramma, 1Cinepyelogram, 236

Decapsulatio renis, 213 Diphallia, 362

Conaden, Strahlenbelastung und Strah-

lenschutz, 230 Gonadi, protezione in radiologia dia-

gnostica, 230

Haemorrhagia renalis et nephrectomia partialis, Ill

Harnblase, Malakoplakie, 350

Harnleiter, Plastik, 193

Harnröhre, operative Behandlung der Strikturen, 374

Hydronephrosis et nephrectomia partialis, Ill

Hyperhydration, akute hypotone, 14

Hyperparathyreoidismus et lithiasis renalis, 294, 317

Induratio penis plastica, 1 Iperparatiroidismo e calculosi renale, 294, 317

Kidney, acute hypotonic hyperhydration, 14

bleeding, partial nephrectomy, Ill

carcinoma 262, 333

denervated, in partial nephrectomy, Ill

hydronephrosis, partial nephrectomy, Ill

osmotic nephrosis, 14

partial nephrectomy, Ill

388 Index rerum ХІП/1962

Pendiomide-injected, Ill

tuberculosis, partial nephrectomy, Ill

Lithiasis renalis 40, 53, 294, 317, 370

Malacoplasia vesical, 350 Monotremization, 254

Nephrectomy, partial, Ill Nephrolithiasis, 40, 53, 294, 317, 370 Nephroptosis, cinepyelogram, 236 Néphrose osmotique, 14 Nervenblock, renaler, Ill Niere, akute hypotone Hyperhydration, 14 Carcinom, Gewebskulturen, 333 -, -, Prognose, 262

Dekapsulation bei Anurie, 213

intrarenaler Druck, 14

osmotische Néphrose, 14

renaler Nervenblock, Ill -, Teilresektion, Ill 
Osmotic anuria, 14

- $\quad$ nephrosis, 14Oxalate calculosis, 370

Partial nephrectomy, Ill Penis duplex, 362

- $\quad$ induratio plastica, 1-, melanoma, 273

-, sarcoma, 273 Peyronie-Disease, 1 Poliomyelitis et urolithiasis, 40 Prostatic cancer, 74, 144

Pseudohermaphroditismus, 65

Radiologia diagnostica, protezione delle

gonadi, 230 Rein, blocage du nerf renal, Ill -, cancer, 262, 333

- decapsulation, 213

-, hyperhydratation hypotonique aigue, 14

-, néphrectomie partielle, Ill

-, néphrose osmotique, 14

-, prognostic du carcinome, 262

-, tuberculose, néphrectomie partielle,

Ill Rene, iperidratazione acuta ipotonica,

14

blocco de П'innervazione renale, Ill

nefrectomia parziale, Ill

Riñón, bloqueo de los nervios renales, Ill

carcinoma, 262, 333

decapsulación, 213

hiperhidratación hipotonica aguda, 14

nefrectomia parcial, Ill Roentgencinematografia delle vie uri-

narie superiori, 236 Roentgen diagnosis, protection of go-nads, 230

Sarcoma penis, 273

Strictures de Гurèthre, traitement opé-ratoire, 374

Tuberculosis renalis et nephrectomia partialis, Ill

Ureter, anastomosis uretero-ureteralis, 193

-, - ileo-coeco-sigmoidea, 254

Urethral stricture, operative management, 374

Urinary bladder, malakoplakia, 350

Urolitiasis, frequentia 53,

hyperparathyreoidismus, 294, 317

nephrectomia partialis, Ill

Oxaluria, 370

P. Poliomyelitis

40

Vescica

malacoplachia

350 X-Rays

protection of gonads

230 